Cahiers de philosophie de l'université de

\title{
Une « mutation » de l'art? Martin Heidegger et Paul Klee
}

\section{Guillaume Fagniez}

\section{(2) OpenEdition}

1 Journals

Édition électronique

URL : https://journals.openedition.org/cpuc/295

DOI : $10.4000 /$ cpuc. 295

ISSN : 2677-6529

Éditeur

Presses universitaires de Caen

\section{Édition imprimée}

Date de publication : 1 novembre 2018

Pagination : 75-94

ISBN : 978-2-84133-904-4

ISSN : 1282-6545

\section{Référence électronique}

Guillaume Fagniez, « Une « mutation » de l'art ? Martin Heidegger et Paul Klee », Cahiers de philosophie de l'université de Caen [En ligne], 55 | 2018, mis en ligne le 01 novembre 2019, consulté le 01 février 2023. URL : http://journals.openedition.org/cpuc/295; DOI : https://doi.org/10.4000/cpuc.295

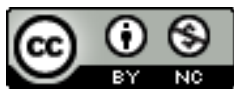

Creative Commons - Attribution - Pas d'Utilisation Commerciale 4.0 International - CC BY-NC 4.0 https://creativecommons.org/licenses/by-nc/4.0/ 


\title{
Une «mutation» de l'art? Martin Heidegger et Paul Klee
}

\begin{abstract}
\ Intérêt de Heidegger pour l'œuvre de certains artistes est bien 1 connu: pour Van Gogh notamment, à travers la conférence sur L'origine de l'œuvre d'art, bien que son nom affleure antérieurement à 1935; pour Cézanne, dont on sait que Heidegger a médité le "chemin ». Mais les noms de bien d'autres artistes jalonnent le parcours de Heidegger, aussi différents que Paula Becker-Modersohn, Georges Braque, Eduardo Chillida. L'un de ces artistes y tient toutefois une place aussi discrète que singulière: le peintre suisse Paul Klee. Discrète, sa présence l'est indéniablement: dans l'œuvre publiée, son nom apparaît rarement, et il semble y avoir en tout et pour tout sept occurrences dans la Gesamtausgabe ${ }^{1}$. On sait notamment que, dans le prologue à la conférence «Temps et être» (1962), deux œuvres de Klee sont mentionnées au titre des quelques objets face auxquels la pensée doit abandonner toute prétention à la compréhension immédiate ${ }^{2}$. Klee est encore nommé dans certains textes plus marginaux, par exemple dans deux entretiens avec le Japonais Shinichi Hisamatsu, datant de mai 1958 et publiés en $2000^{3}$.

L'importance de Klee pour Heidegger paraît ainsi à première vue peu attestée par l'œuvre elle-même. Elle est en revanche bien documentée à travers deux sources. D'abord un ensemble de notes prises par Heidegger sur Klee, 17 petits feuillets composés de passages de textes de Klee recopiés par le penseur, d'esquisses de sa main reprenant certains tableaux, de quelques bribes de réflexions sur les œuvres en question. Ces notes, datant
\end{abstract}

1. Voir M. Heidegger, «Zeit und Sein», in GA 14, 5 ; «Die Kunst und das Denken », in GA 16, 556; «Wechselseitige Spiegelung», in GA 16, 776-779; "Bild und Wort», in GA 74, 185; "Kunst und Technik», in GA 76, 384.

2. M. Heidegger, «Zeit und Sein», in GA 14, 5; trad. fr.: «Temps et être», F. Fédier (trad.), in Questions IV, Paris, Gallimard, 1976, p. 12.

3. M. Heidegger, «Die Kunst und das Denken» et «Wechselseitige Spiegelung», in GA 16, 552-557 et 776-78o. Voir, dans le présent numéro, p. 11-16 et 17-20. 
vraisemblablement des années 1950, ont été décrites en 1993 par Günter Seubold ${ }^{4}$, qui a cherché en vain dans le fonds posthume une conférence sur Klee dont on a longtemps cru qu'elle avait été tenue en $1956^{5}$.

La seconde source est un texte qui n'est pas de la main de Heidegger, mais de son ami Heinrich Wiegand Petzet, dans son livre de souvenirs sur Heidegger intitulé Auf einen Stern zugehen ${ }^{6}$. Petzet est un historien de l'art. C'est lui qui conduit Heidegger en 1930 à Worpswede, une colonie d'artistes située non loin de Brême, où avait en particulier été active Paula BeckerModersohn; c'est lui encore qui emmène Heidegger voir les Van Gogh du Kröller-Müller Museum aux Pays-Bas; c'est par son entremise enfin que Heidegger peut découvrir, en 1959, la collection Klee de l'industriel américain Thompson, provisoirement déposée par la galerie Beyeler de Bâle dans une maison patricienne d'un faubourg de cette même ville.

D’après le témoignage de Petzet, Heidegger contempla de longues heures - en fait presque toute une journée - les 88 œuvres de Klee exposées dans cette maison ${ }^{7}$. Heidegger connaissait l'œuvre de Klee: celle-ci avait acquis une notoriété internationale dès le début des années 1920. Des conversations au sujet du peintre avaient déjà eu lieu entre Heidegger et Petzet, qui avait une connaissance intime de l'œuvre de Klee ${ }^{8}$. Les notes sur Klee sont probablement antérieures à 1959. Toujours est-il que cette visite marque, à en croire Petzet, le point culminant de la rencontre avec Klee, à partir duquel l'œuvre de ce dernier trouve véritablement une résonance dans la pensée de Heidegger.

Petzet a consigné dans ses souvenirs les quelques œuvres de Klee auxquelles Heidegger a été particulièrement sensible. Il en cite sept ${ }^{9}$, que

4. G. Seubold, «Heideggers nachgelassene Klee-Notizen», Études heideggeriennes, vol. 9, 1993, p. 5-12.

5. Elle figure notamment dans la chronologie établie par W. Biemel, Heidegger, Hambourg, Rowohlt, 1973, p. 154.

6. H. W. Petzet, Aufeinen Stern zugehen. Begegnungen und Gespräche mit Martin Heidegger, 1929 bis 1976, Francfort-sur-le-Main, Societäts-Verlag, 1983; Le chemin de l'étoile. Rencontres et causeries avec Heidegger, 1929-1976, C.-N. Grimbert, P. Arjakovsky (trad.), Paris, Éditions du Grand Est, 2014.

7. La collection ayant été intégralement acquise par le musée de Düsseldorf, il est possible d'en prendre connaissance en se reportant à l'ouvrage de W. Schmalenbach, Paul Klee. Die Düsseldorfer Sammlung, Munich, Prestel-Verlag, 1986. Beyeler n'a conservé qu'une seule toile (Ein Tor), justement «en souvenir de l'émotion éprouvée par le penseur " (voir H. W. Petzet, Le chemin de l'étoile..., p. 172).

8. Non seulement Petzet était un proche de Georg Schmidt, directeur du musée des Beaux-Arts de Bâle de 1936 à 1961, mais il avait en outre préparé l'édition des lettres de Rilke à Klee. Felix Klee, fils de Paul, lui avait fait longuement visiter la succession Klee dans son appartement de Berne (voir H. W. Petzet, Le chemin de l'étoile..., p. 170).

9. Radiodymmolatoren (titre donné par Petzet: sans doute Überkultur von Dynamoradiolaren, dessins de 1926), Der Beladene (1929), Gedanken bei Schnee (1933), Patientin (1933), Heroische Rosen (1938), Ein Tor (1939), Heilige, aus einem Fenster (1940). 
l'on peut également rapprocher des dix œuvres mentionnées dans les notes ${ }^{10}$. La plupart ont en commun de dater de la deuxième moitié des années 1930, période pendant laquelle les œuvres de Klee sont traversées par l'angoisse et la mort. À partir de 1935, Klee est malade; il meurt en 1940. Heidegger cite dans ses notes la toute dernière toile peinte par Klee, Mort et $f e u$. Et Petzet rapporte que Heidegger a été frappé par la gouache intitulée Une porte: "C'est, dit Heidegger, la porte par laquelle nous devons tous passer un jour - la mort ${ }^{11}$. On connaît le caractère central de la finitude de l'existence dans la pensée de Heidegger: rien d'étonnant, à première vue, à ce que ces œuvres trouvent un écho particulier chez le philosophe. Dans le même ordre d'idées, on peut relever la foudre de Éclair multicolore (1927) et Combat harmonisé (1940): on a là deux phénomènes dont l'image est lestée d'un contenu philosophique depuis les tout premiers penseurs; et l'on peut penser aux fragments d'Héraclite sur la foudre (fragment 64) et sur le polemos (fragment 53) commentés à de nombreuses reprises par Heidegger, entre autres - mais pas seulement - dans le séminaire de 1966-1967 avec Fink ${ }^{12}$. Au premier abord, Heidegger paraît retenir des œuvres chargées de sens philosophique et prêter moins d'attention à leur aspect proprement plastique ${ }^{13}$.

Un tel constat n'est toutefois pas tout à fait juste, puisque toute une part des toiles citées par Heidegger n'est guère porteuse d'un «sujet signifiant», ou d'un thème philosophique: voir par exemple Sainte, à une fenêtre (1940), Roses héroïques (1938) ou Pensées par temps de neige (1933). Mais surtout, on risque fort, en supposant que Heidegger cherche dans l'œuvre de Klee l'illustration de thèses philosophiques, de manquer l'essentiel de la rencontre. Ce dernier paraît résider dans le fait que Heidegger aborde l'œuvre du peintre au premier chef dans la perspective de "l'histoire de l'être», et de l'histoire de l'art elle-même envisagée à partir de cette perspective. Sous cet horizon historique - ou «historial»-, Heidegger avance deux idées qui, pour le moins, requièrent une explication. Tout d'abord une double thèse concernant la situation historique de l'art: 1) Heidegger considère

10. Der Gott des nördlichen Waldes (1922), Kleine Felsenstadt (1932), Ruhende Sphinx (1934), Büsser (1935), Harmonisierter Kampf (1937), Gesicht einer Gegend (1938), Ernste Miene (1939), Heilige, aus einem Fenster (1940), Hoher Wächter (1940), Tod und Feuer (1940).

11. H. W. Petzet, Le chemin de l'étoile..., p. 171-172.

12. Sur le fragment 53, voir par exemple le cours de 1933-1934, "Vom Wesen der Wahrheit», in GA 36/37, 89 sqq.

13. Voir notamment T. Hildebrandt, " "Bildnerisches Denken”. Martin Heidegger und die bildende Kunst», in Heideggers Ursprung des Kunstwerks. Ein kooperativer Kommentar, D. Espinet, T. Keiling (dir.), Francfort-sur-le-Main, V. Klostermann, 2011, en particulier p. 215; dans le même sens, S. H. Watson, «Heidegger, Paul Klee, and the Origin of the Work of Art», Review of Metaphysics, vol. 6o, n² 2, 2006, p. 327-357. 
que l'art moderne dans son ensemble est «d'essence métaphysique ${ }^{14}$; 2) par rapport à ce déploiement métaphysique de l'art, Cézanne aurait «préparé» une «mutation [Wandlung] » de l'art qui aurait été véritablement «accomplie» par Klee ${ }^{15}$. D'où un premier massif de questions à défricher, au fil de l'analogie qui se dessine entre histoire de la métaphysique et histoire de l'art: en quoi l'art se serait-il déployé sur un mode métaphysique? En quoi y aurait-il, suivant toujours cette hypothétique analogie, «fin de l'art» et «dépassement de l'art» - comme il y a «fin» et «dépassement de la métaphysique»? Quel rôle revient à Klee dans un tel tournant de l'art?

Deuxième idée, avancée dans des propos recueillis par Petzet, et qui concerne cette fois l'auto-interprétation de Heidegger: «Il lui faudrait, à lui Heidegger [suite à cette rencontre avec l'œuvre de Klee], écrire une deuxième partie de L'origine de l'œuvre d'art! ${ }^{16}$. Au-delà des spéculations sur le contenu d'une telle deuxième partie, cette idée invite à interroger le sens de la conférence de Francfort à partir de l'interprétation rétrospective de Heidegger lui-même, et ainsi à souligner fortement le statut intermédiaire - et donc quelque peu précaire - du texte dans l'œuvre de Heidegger. On comprend bien en effet qu'une telle «deuxième partie» ne pourrait simplement venir "compléter» la conférence en s'additionnant à ce qui serait alors une «première partie». $\mathrm{Si}$ «mutation» ou «tournant» de l'art il y a, la conférence se trouve dès lors exposée sous un angle nouveau: en quel sens la conférence se voit-elle mise en cause? Dans quelle mesure au contraire ses thèses devraient-elles être maintenues?

\section{Essence métaphysique de l'art et essence technique de la métaphysique: le Bild}

Tout d'abord, en quoi l'art moderne est-il d'essence métaphysique? D'après les textes de Heidegger des années 1950, non seulement l'art moderne, mais l'art occidental dans son ensemble, est métaphysique en tant qu'il est conçu à partir de l'image, du Bild. Et c'est en se soustrayant à une telle perspective que Klee s'affranchirait du même coup d'une tradition métaphysique de l'art. Cette indication est donnée notamment dans l'entretien du 18 mai 1958

14. Voir H. W. Petzet, Le chemin de l'étoile..., p. 169 et G. Seubold, «Heideggers nachgelassene Klee-Notizen », p. 10.

15. Selon un autre énoncé, cette mutation aurait été "préparée» chez Cézanne, mais rendue «manifeste» chez Klee (voir H. W. Petzet, Le chemin de l'étoile..., p. 169).

16. Voir H. W. Petzet, Le chemin de l'étoile..., p. 172, ainsi que le témoignage de O. Pöggeler dans "Heidegger und Klee - Überlegungen zur Kunst", in Kunst und Wahrheit. Festschrift für Walter Biemel zu seinem 85. Geburtstag, M. Diaconu (dir.), Bucarest, Humanitas, 2003, p. 202. 
avec Hisamatsu. Heidegger y met en contraste «l'art européen » caractérisé par la «représentation [Darstellung] », c'est-à-dire par l'image ou la figure en tant qu'elle rend visible [das sichtbarmachende Bild], et l'art zen, dont le propre serait d'entrer en rapport immédiat avec l'origine même des choses: l'essence de l'art zen, c'est, dans les termes d'Hisamatsu, le «jaillissement de l'origine ${ }^{17}$. Au cours de l'entretien, le nom de Paul Klee est prononcé; Heidegger refuse aussitôt l'assignation de ce dernier au Bild, et laisse entendre que l'œuvre de Klee serait à situer plutôt du côté de l'art zen. Petzet le rapporte également, Heidegger dit des œuvres de Klee qu'elles sont «non pas des images, mais des états [nicht Bilder, sondern Zustände]» qui "font "voir" des tonalités affectives [Stimmungen] ${ }^{18}$. Klee, ainsi rapproché de l'Extrême-Orient - qui représente aux yeux de Heidegger une sorte d'«en dehors» de la métaphysique -, paraît donc bénéficier d'un statut d'exception qui le situerait en un «au-delà» de la métaphysique.

Pour le comprendre, concentrons-nous sur le Bild comme marqueur du caractère métaphysique de l'art. En quoi la conception de l'art au prisme de l'image relève-t-elle de la métaphysique? Le cours du semestre d'été 1942 sur l'hymne de Hölderlin L'Ister permet d'éclairer cette question. Au début du cours, Heidegger explique brièvement ce qu'il appelle «l'interprétation métaphysique de l'art», dans un contexte où il récuse une lecture symbolique du fleuve: l'Ister, c'est-à-dire le Danube, ne « représente » pas dans le poème quoi que ce soit d'autre que lui-même, et il n'est chargé de rendre sensible aucune idée. Le dualisme sous-jacent à une telle conception - entre le «sensible» et ce qui n'est visible que du seul « œil de l'esprit» - est reconduit par Heidegger à celui propre à la conception platonicienne de l'être, divisé en to aisthêton et to noeton. Tout l'art occidental reposerait dès lors sur ce clivage et pourrait être envisagé comme une matière porteuse d'une forme ou d'une idée suprasensible, en tout état de cause d'un point de référence eidétique. Ce dualisme structurel de l'art, caractéristique de la métaphysique, conduit Heidegger à énoncer deux thèses. Premièrement, l'articulation du Sinn et du Bild au sein de l'œuvre d'art subit une série de transformations qui correspondent chaque fois à la manière dont l'homme se rapporte au monde:

[...] la peinture sur vase grecque, les peintures murales de Pompéi, les fresques de Reichenau de l'époque ottonienne, les peintures de Giotto, une peinture de Dürer et un tableau de Caspar David Friedrich ne sont pas seulement différents du point de vue de leur style, mais le style lui-même est chaque fois différent

17. Voir M. Heidegger, «Die Kunst und das Denken», in GA 16, 553-554.

18. Voir H. W. Petzet, Le chemin de l'étoile..., p. 174. 
du point de vue de son essence métaphysique. Ce qui s'appelle « réalité» dans la représentation de l'Ancolie de Dürer est déterminé autrement que le « réel» dans une fresque médiévale. Plus exactement: les deux œuvres d'art donnent du réel une apparition figurée où le sens de la réalité est chaque fois différent ${ }^{19}$.

Le «sens de la réalité », c'est-à-dire ce qui est chaque fois tenu pour l'être de l'étant: un statut ontologique est ici accordé au style artistique, et l'on est tenté de dire, en paraphrasant Buffon : «le style est l'être même ${ }^{20}$. Dans les termes du Heidegger des années 1930, et en particulier de la conférence de Francfort: la manière dont le monde s'ouvre chaque fois à une humanité historique s'inscrit dans l'œuvre. L'œuvre d'art «met en œuvre» la "vérité» et du même coup atteste la particularité d'un monde historique. Ainsi, il y aurait bien consonance entre histoire de l'être et histoire de l'art, cette dernière recueillant à sa façon propre les différentes manières, pour la vérité, d' «avoir lieu» ${ }^{21}$.

Venons-en à la deuxième thèse, la plus fondamentale: "Mais - ajoute Heidegger - ces différentes formes de l'essence de la réalité se tiennent pourtant toujours au sein des lignes fondamentales de l'articulation métaphysique du monde ${ }^{22}$. Aussi longtemps que l'art relève du Bild, qu'il se comprend lui-même comme rapport de deux côtés, sensible et suprasensible, il demeure l'expression d'une lecture métaphysique du monde. On le voit, l'image n'est pas ici une catégorie esthétique, mais plutôt une détermination seinsgeschichtlich, qui marque tout à la fois l'appartenance de l'art à la métaphysique, et l'inclusion de l'histoire de l'art au sein de l'histoire de l'être.

La question se pose pourtant de savoir si la conception de l'art à l'horizon du Bild reconduit simplement le dualisme structurel de la métaphysique, et si l'art est seulement une expression parmi d'autres du déploiement métaphysique de l'histoire occidentale. Or tel n'est pas le cas, loin s'en faut. Pourquoi? Il faut d'abord rappeler que le terme allemand Bild - à la différence de l'«image» qui renvoie originellement à l'idée d'imitation ${ }^{23}$ - implique

19. M. Heidegger, Hölderlins Hymne "Der Ister", GA 53, 28. (Les fresques de l'église SaintPierre et Saint-Paul, dans l'île monastique de Reichenau sur le lac de Constance, datent du Xe $\mathrm{X}^{\mathrm{e}}$ siècle.)

20. Buffon dit que «le style est l'homme même» (Discours de réception à l'Académie française, 1753).

21. Voir, en ce sens, la remarque de Philippe Descola selon laquelle le passage à ce qu'il appelle le «naturalisme» aurait été attesté dans les images avant d'être explicité dans le discours (La composition des mondes. Entretiens avec Pierre Charbonnier, Paris, Flammarion, 2014, p. 254 sq. et 272 sq.).

22. M. Heidegger, GA 53, 28.

23. Imago, c'est la représentation, le portrait, la réplique: la statue est «image» de l'ancêtre défunt, le fils «image» du père, etc. Le mot est de même racine que le verbe imitor, imiter, reproduire par imitation. 
l'idée de la production d'une figure ou d'une forme. C'est à ce titre qu'il est non seulement un phénomène frappé du sceau de la métaphysique parmi d'autres, mais en outre un concept clef de la description heideggerienne de l'histoire de la métaphysique, puisqu'il en marque le commencement et la fin. L'art, au sens de la technè, avec son eidétique propre, joue en effet un rôle décisif dans la fondation de l'histoire de la métaphysique: les premières interprétations grecques de l'être, notamment chez Aristote, se règlent, selon Heidegger, sur le savoir technique, sur la figure que vise tout acte de production. Mais le Bild est tout particulièrement présent dans l'«achèvement de la métaphysique» sous la forme d'une «métaphysique de la subjectivité». Dans ce contexte, le Bild reçoit un sens relevant pleinement de l'«histoire de l'être», notamment dans la conférence de 1938 «Die Zeit des Weltbildes», qui envisage comme les deux faces d'un seul et même phénomène fondamental de l'époque moderne «le devenir-image du monde et le devenir-sujet de l'homme [das Bild-werden der Welt und das Subjekt-werden des Menschen] $»^{24}$. Ce phénomène procéderait de la mutation de la vérité en certitude, par laquelle l'homme-sujet se tient désormais au fondement de la vérité de toute chose ${ }^{25}$. Lorsque la vérité devient certitude et l'homme sujet, le monde n'est rien d'autre que Bild, corrélat de ce sujet. L'insuffisance de la traduction de Bild par «image» est manifeste, puisqu'il ne s'agit pas tant ici d'une dérivation et d'une déperdition ontologique - la copie étant moins réelle que l'original - que d'une subordination du monde à la subjectivité en tant qu'elle se le représente et le produit: «Le mot Bild signifie à présent: la configuration de la production par représentation [das Gebild des vorstellenden Herstellens] ». Il caractérise le monde en tant qu'il est intégralement «établi par l'homme qui se représente et produit [sofern es durch den vorstellend-herstellenden Menschen gestellt ist $\rrbracket_{{ }^{26}}$. Le Bild, en tant qu'il désigne un monde intégralement établi par l'homme, introduit ainsi à ce que Heidegger va bientôt appeler le «Gestell» - ce mode d'être fonctionnant comme un doublet négatif de l'événement, selon lequel l'unité et l'entièreté d'un monde procèdent intégralement d'une instance subjective, si bien qu'il n'y a plus de monde au sens d'une entrée en présence à partir de soi.

Ainsi, d'un côté - selon le cours de 1942 -, le Bild relève d'une «interprétation métaphysique de l'art». D’un autre côté - d'après la conférence de 1938 -, la métaphysique elle-même peut être conçue à partir du Bild. Or,

24. M. Heidegger, "Aus dem Umkreis der Besinnung auf die Neuzeit (Der Übergang)», in GA 76, 91. Voir «Die Zeit des Weltbildes", in GA 5, 92; trad. fr.: «L'époque des conceptions du monde», in Chemins qui ne mènent nulle part, W. Brokmeier (trad.), Paris, Gallimard, 1962, p. 120-121.

25. Voir M. Heidegger, GA 5, 88; Chemins..., p. 115.

26. M. Heidegger, GA 5, 94 et 89; Chemins..., p. 123 et 117. 
dès lors que le Bild, et avec lui l'art européen, apparaît comme métaphysique de part en part, la question ne manque pas de se poser: quel peut être le statut de l'œuvre d'art dans le contexte de l'achèvement de la métaphysique, sinon celui d'une simple dimension de l'autoréflexion du sujet, une mise en abyme du Bild? Une alternative semble ici s'imposer qui est aussi une sorte de bifurcation «historiale». Soit l'art relève par principe et irrémédiablement du Bild, et dès lors il est "metaphysisch am Ende ${ }^{27}$ : en tant qu'il est métaphysique, il touche à sa fin. Soit il y a, au-delà du Bild, une possibilité non métaphysique de l'art, une possibilité de re-commencer l'art sous un tout autre régime que celui du Bild: on reconnaît ici le thème du « dépassement de la métaphysique» et la question d'un "autre commencement», placés sur le terrain de l'art ${ }^{28}$.

Cette question de la fin de l'art est en même temps celle du statut de la conférence sur l'œuvre, dont Heidegger lui-même s'est demandé quelle "place» lui revenait "au sein du dépassement de la métaphysique ${ }^{29}$. Appartient-elle au champ ouvert par le «premier commencement»? Si tel était le cas, son propos serait au moins en partie caduc, son concept d'art périmé aussitôt qu'introduit. Prépare-t-elle au contraire, à sa manière, un "autre commencement», celui de l'art - et peut-être au-delà? Tentons d'éclaircir la première question, celle de la fin de l'art, en partant de la seconde, celle de la péremption de la définition de l'art dans la conférence de Francfort.

\section{La fin de l'art}

Heidegger évoque explicitement, à plusieurs reprises, la «fin de l'art», en se référant chaque fois à la fameuse thèse de Hegel selon laquelle l'art serait "chose du passé » ${ }^{30}$. La formule de Hegel est bien évidemment relue à l'aune de l'histoire de l'être: il s'agit de la fin de l'art en tant qu'il est métaphysique. Commençons par entendre ce propos sur la «fin de l'art» comme une sorte de palinodie à l'égard de la conférence sur l'œuvre, une mise en cause de la validité de son concept d'art en tant que «mise en œuvre de la vérité»

27. M. Heidegger, «Kunst und Technik», in GA 76, 377.

28. Cette interrogation de «l'autre de l'art» est notamment formulée dans Überlegungen VIII, GA 95, 134-137.

29. M. Heidegger, «Die Überwindung der Metaphysik», in GA 67, 109.

30. Cette idée d'une fin de l'art rapportée à Hegel apparaît en plusieurs occurrences : la postface à L'origine de l'œuvre d'art (GA 5, 67 sqq.) ; des notes de 1938-1939 liées à la conférence sur l'œuvre d'art («Kunst und Metaphysik», in GA 67, 107 sqq.); des notes contemporaines de la conférence sur l'essence de la technique («Kunst und Technik», in GA 76, 376-378); enfin dans une lettre à Petzet de 1972 (voir H. W. Petzet, Le chemin de l'étoile..., p. 162-163). 
et «installation du monde». Dans la conférence de Francfort, la nécessité de l'œuvre d'art est fondée sur la vérité, plus exactement sur la structure même de la vérité, de sorte que s'y enracine une «aspiration à l'œuvre», une «attraction vers l'œuvre [Zug zum Werk]» soulignée avec insistance par Heidegger ${ }^{31}$. Ce qui rend nécessaire l'œuvre d'art, c'est, schématiquement, le conflit inhérent à la vérité, entre déploiement et non-déploiement de l'être: la vérité nécessite l'œuvre pour y trouver son espace d'ouverture et de déploiement. Mais, à l'âge de la métaphysique achevée - c'est-à-dire à l'âge du déploiement total de l'étant à partir d'un fondement subjectif -, ce conflit du retrait et du non-retrait est arbitré par avance dans le sens de la translucidité de l'étant pour l'instance représentante et productrice dont il procède. Dès lors, une «mise en œuvre de la vérité» paraît à tout le moins superflue: à la nécessité de l'art soulignée par la conférence, l'âge de la métaphysique achevée oppose son impossibilité. C'est pourquoi Heidegger est amené à constater, dès 1938-1939, que - dès lors qu'il n'y a d'art qu' «au sein de la métaphysique et avec elle» - «avec la fin de la métaphysique [se produit] la fin de l'art», c'est-à-dire la fin de la possibilité «qu'une œuvre puisse par elle-même fonder la vérité ${ }^{32}$. En d'autres termes encore: l'humanité propre à l'âge de la métaphysique produit une totalité organisée au sein de laquelle une «institution artistique du monde» se trouve toujours déjà pour ainsi dire prévenue, et sans objet. Au sein du "dispositif» par lequel toute chose est mise au service du sujet-homme, l'installation d'un monde ne répond à aucune nécessité: l'art, comme Aufstellen der Welt, «se dissout» dans le Gestell 33, il y est intégré et neutralisé sous la forme spectrale de la production culturelle ${ }^{34}$. C'est en ce sens que Heidegger, dans une lettre à Petzet de 1972, évoque «la fin de l'art, d'un art instituant le monde [Ende der weltstiftenden Kunst], à l'âge de la dissolution de la métaphysique en une technologie universelle ${ }^{35}$.

Voilà qui offre un éclairage plausible quant à la nécessité d'écrire une «seconde partie» de la conférence sur l'œuvre d'art: tout son propos serait miné par un fâcheux contretemps, puisqu'elle fonde la nécessité de l'œuvre sur une situation historique dépassée par l'achèvement de la métaphysique. Il conviendrait donc désormais - telle est notamment la lecture d'Otto Pöggeler - de formuler une caractérisation de l'art «à l'âge de la technique».

31. Voir M. Heidegger, GA 5, 44, 48, et 50; Chemins..., p. 63, p. 67 et p. 69.

32. M. Heidegger, "Kunst und Metaphysik», in GA 67, 108.

33. M. Heidegger, «Kunst und Technik», in GA 76, 377.

34. Dans son important $\$ 11$, le traité Besinnung distingue en ce sens l'art symbolique, esthétique et métaphysique, et l'art comme mode d'«installation» de l'étant propre à la Machenschaft (voir GA 66, 36-37).

35. H. W. Petzet, Le chemin de l'étoile..., p. 163. 
Or Klee, artiste répondant très consciemment, dans son art, à un monde bouleversé par le phénomène de la technique, pourrait inspirer une telle caractérisation $^{36}$.

Cette lecture, assurément possible, ne creuse pas encore, semble-t-il, le problème à fond. Car il y a un second motif de palinodie, qui est l'inscription de la métaphysique au cœur même de la conférence. Il faut rappeler en effet la situation intermédiaire de la conférence au sein de l'œuvre de Heidegger, dès lors qu'elle se trouve pour ainsi dire dans la trajectoire de la phase «métaphysique et transcendantale» de sa pensée. À partir de Sein und Zeit, Heidegger développe provisoirement ce qu'il appelle une «métaphysique du Dasein ", c'est-à-dire une pensée de l'existence à partir de sa transcendance. Or cette transcendance - cet élan de l'existence qui la porte, au-delà des choses qu'elle rencontre, vers le monde qui est l'horizon de leur apparition - a un double sens, à la fois passif et actif, réceptif et spontané. Ce second aspect est particulièrement accentué par Heidegger après Sein und Zeit: le monde m'advient, mais cet «avoir-lieu» du monde est tout à la fois une configuration du monde, une Weltbildung qui relève du projet de l'existence ${ }^{37}$. Or, la thèse de l'installation artistique d'un monde peut être envisagée comme aboutissement de celle du caractère «configurateur de monde» de l'existence. De la même façon, la vérité - qui donne tout son sens à l'œuvre -, si elle est manifestation primordiale, est également un non-retrait conquis par l'existence dans une forme de lutte. Si bien que la conférence, en identifiant le Geschehen de la vérité dans l'œuvre à une «mise en œuvre de la vérité [Ins-Werk-Setzen der Wahrheit]», hérite largement du caractère «actif» propre à la transcendance. Dès lors, il est permis de se demander si la conférence de Francfort ne serait pas elle aussi frappée par la «rechute dans la métaphysique ${ }^{38}$ que Heidegger a diagnostiquée dans son propre parcours. Cela éclairerait en tout état de cause le propos de Heidegger qui, dans des notes de 1938-1939, voit dans la conférence « une

36. Voir O. Pöggeler, «Heidegger und Klee - Überlegungen zur Kunst», p. 202. Pour Pöggeler, la grande différence entre les années 1930 et les années 1950, c’est que Heidegger interroge désormais l'art dans son rapport à la technique. S'agissant de Klee, il faut remarquer que son œuvre est postérieure à la Première Guerre mondiale, et que sa Schöpferische Konfession notamment se concentre sur le rapport de l'art au monde moderne (voir O. Pöggeler, "Über die moderne Kunst". Heidegger und Klee's Jenaer Rede von 1924, Erlangen - Iéna, Verlag Palm \& Enke, 1995, p. 1 et 5 sqq.).

37. Voir notamment M. Heidegger, Die Grundbegriffe der Metaphysik. Welt - Endlichkeit Einsamkeit, GA 29/30, 509; trad. fr.: Les concepts fondamentaux de la métaphysique. Monde - finitude - solitude, D. Panis (trad.), Paris, Gallimard, 1992, p. 504.

38. M. Heidegger, "Die Überwindung der Metaphysik», in GA 67, 63: "En rester à la "transcendance", même interprétée de façon plus originale à partir du Da-sein, est une rechute [Rückfall] dans la métaphysique». 
tentative de sauver l'art par une interprétation plus originale de l'œuvre d'art» - tentative de sauvetage qualifiée par lui d' "erronée [irreführend] »39.

C'est justement parce que règne une «ambivalence [Zwiespältigkeit]» ${ }^{40}$ dans la conférence que l'œuvre de Klee est appelée à venir en infléchir le sens. Force est de constater toutefois que l'art moderne en général - mais également celui de Klee dans une certaine mesure - peut être interprété dans le sens de son appartenance à la «fin de la métaphysique». Très schématiquement, on peut distinguer deux moments attestant cette appartenance. Tout d'abord un subjectivisme: Malraux, dans des pages bien connues des Voix du silence, met en évidence le changement de la relation de l'artiste à la nature: «ce que cherchait le nouvel art, c'était le renversement de la relation entre l'objet et le tableau, la subordination de l'objet au tableau ${ }^{41}$. Tandis que naguère l'art avait toujours conservé, peu ou prou, une dimension mimétique de représentation, au sein de laquelle la nature faisait figure de fin et l'art de moyen, pour le peintre moderne

l'univers deviendra le moyen éclatant de son propre langage. La fin dont l'acuité de la vision n'est que le moyen, c'est la transformation des choses en un univers plastique autonome, cohérent et particulier. [...] À la représentation du monde succède son annexion. [...] l'art moderne [...] est l'annexion des formes à un schème intérieur qui prend ou non forme de figures ou d'objets, mais dont figures et objets ne sont que l'expression. La volonté initiale de l'artiste moderne c'est de tout soumettre à son style, et d'abord l'objet le plus brut, le plus nu. Son symbole, c'est la Chaise de Van Gogh... la chaise isolée (avec à peine une suggestion de misérable repos) comme un idéogramme du nom même de Van Gogh ${ }^{42} \ldots$

L'art moderne porterait donc à son comble l'idée du style, entendue comme un principe d'appropriation et de domination du monde. Et dès lors, il apparaît comme une expression de la métaphysique de la subjectivité: le style, c'est l'être, mais ce dernier ne diffère plus désormais de l'homme ${ }^{43}$. Nous sommes ainsi conduits au second moment, celui du constructivisme. L'art moderne, en explicitant un principe subjectif qui a du reste peut-être

39. M. Heidegger, GA 67, 107 .

40. Ibid.

41. A. Malraux, Les voix du silence, Paris, Gallimard, 1951, p. 115.

42. Ibid., p. 117.

43. Il est vrai qu'à cette lecture de Malraux - et à l'usage qu'on peut en faire dans le cadre de l'histoire de l'être - on serait en droit d'opposer l'interrogation de Merleau-Ponty: toute représentation n'est-elle pas déjà «métamorphose»? L'art moderne, dans son "passage au subjectif", ne met-il pas simplement en lumière une vérité sur laquelle repose toute l'histoire de l'art? (voir M. Merleau-Ponty, «Le langage indirect et les voix du silence», in Signes, Paris, Gallimard, 1960, p. 60 sqq.). 
été sous-jacent à toute l'histoire de l'art, affirme de façon caractéristique l'autonomie de l'œuvre et avec elle l'idée d'une construction interne affranchie de toute description de la nature. Cet aspect, si manifestement présent chez Picasso, est explicitement revendiqué par Klee. Sans doute cela explique-t-il en partie le jugement négatif porté par Heidegger sur les textes du Klee théoricien. Dans un important compte rendu d'exposition de 1912, Klee décrit l'expressionnisme - et le cubisme comme un cas particulier de celui-ci - à partir de ce qu'il appelle «la construction active de la forme». Heidegger recopie entièrement cette description, dans laquelle Klee insiste sur la «violence» que les objets doivent subir pour être «intégrés» à «l'armature» du tableau, et sur leur soumission inconditionnelle à une «loi propre à l'art»:

Pourtant, c'est la représentation détachée de la nature qui redevient la norme, et ainsi la construction [Konstruktion] acquiert une importance accrue, y compris à titre d'expédient technique. Ainsi, c'est l'armature de l'organisme du tableau [das Gerüst des Bildorganismus] qui vient au premier plan, et qui devient la vérité coûte que coûte. Pour s'intégrer à une armature intéressante, les maisons se mettent à pencher [...], violence est faite aux arbres, les humains ne sont plus en état de vivre, l'objet devient méconnaissable au point que l'on croit à une mystification [bis zum Vexierbild]. C'est qu'ici ne règne nulle loi profane, mais au contraire une loi propre à l'art. Dans le tableau, les maisons penchées ne tombent pas, les arbres n'ont pas besoin de refleurir ni les hommes de respirer. Les tableaux ne sont pas des tableaux vivants [Bilder sind keine lebenden Bilder] ${ }^{44}$.

Klee relève l'«inconséquence» qu'il y a à provoquer la «destruction par amour de la construction». Il reconnaît cependant une certaine légitimité à l'«abandon de l'objet [Weglassung des Gegenstandes]» au profit de ce qu'il appelle un «art pur», justement pour autant qu'il parvient à insuffler au tableau une "vie plastique». Ce dernier acquiert ainsi, comme chez Delaunay et Kandinsky, une véritable autonomie, et «mène une existence tout à fait abstraite, sans motif tiré de la nature» ${ }^{45}$.

\section{Paul Klee: un autre commencement de l'art?}

Qu'en est-il de la démarche propre à Klee lui-même? Elle repose justement sur une problématisation explicite de l'antagonisme et de la tension entre

44. P. Klee, «Die Ausstellung des Modernen Bundes im Kunsthaus Zürich» [juillet 1912], in Schriften. Rezensionen und Aufsätze, C. Geelhaar (éd.), Cologne, DuMont, 1976, p. 106-107; trad. fr.: «Approches de l'art moderne», in Théorie de l'art moderne, P.-H. Gonthier (éd. et trad.), Paris, Denoël - Gallimard, 1998, p. 10-11.

45. P. Klee, «Die Ausstellung des Modernen Bundes... », p. 108; «Approches de l'art moderne», p. 12-13. 
l'autonomie de l'œuvre et le rapport de l'art à la nature ${ }^{46}$. Comment l'art peut-il satisfaire à ses propres tâches, accomplir sa vocation propre - qui relève de la "construction", ou plutôt de la "composition" plastique ${ }^{47}$ sans pour autant se refermer sur lui-même et tourner le dos à la nature? Comment peut-il à la fois "renoncer» et "acquiescer » à l'objet? Telles sont les questions qui conduisent Klee à tenter d'ouvrir une voie médiane entre les deux pôles de l'art et de la nature: l'artiste, écrit-il, ne doit être «ni serviteur soumis, ni maître absolu, mais simplement intermédiaire [weder dienen noch herrschen, nur vermitteln] ${ }^{48}$, il doit instaurer un véritable «dialogue avec la nature», un «dialogue avec l'objet». C'est cette voie qui permet à Klee d'être un peintre de l'œuvre autonome et simultanément un peintre des choses - et même d'être "le plus grand réaliste de ce temps" (Georg Schmidt) ${ }^{49}$. Et ce sont les solutions originales trouvées par Klee à cette forme moderne du problème du rapport de l'art à la nature qui vont le conduire, dans des textes de 1923 et $1924^{50}$, à des énoncés proches de ceux de Heidegger. Les moyens conceptuels d'une solution à cette contradiction - entre construction et description -, Klee les trouve en effet dans le couple Terre-Monde, celui-là même qui, chez Heidegger, fait passer de l'analyse de l'œuvre - la conférence de Francfort de 1935-1936 - à celle de la «chose» en tant que telle - la conférence de Brême de 1949 -, c'est-à-dire également d'une pensée encore partiellement imprégnée de schèmes transcendantaux à une pensée du monde lui-même, expressément détaché du pouvoir fondateur et constituant de la subjectivité.

Que dit Klee? Pour concilier les deux impératifs contradictoires de l'art et de la nature, il faut parvenir à dépasser leur différence, et notamment dépasser l'extériorité optique qui en constitue la dimension fondamentale. C'est pourquoi la conférence d'Iéna évoque le «regard pénétrant [durchdringendes Blick]» de l'artiste, qui perce les «apparences de la nature», ses «formes arrêtées [Form-Ende]", pour s'attacher à suivre «l'essence du processus créateur dans la nature», et les «forces formatrices [formende Kräfte]» de

46. Voir en particulier D. Kahnweiler, «À propos d'une conférence de Paul Klee» et «La montée du cubisme», in Confessions esthétiques, D. Naville (trad.), Paris, Gallimard, 1963, respectivement p. 168-179, et p. 9-60.

47. Voir P. Klee, «Übersicht und Orientierung auf dem Gebiet der bildernischen Mittel und ihre räumliche Ordnung», in Das bildnerische Denken, J. Spiller (éd.), Bâle, Schwabe, 1971, p. 92 ; trad. fr.: «De l'art moderne", in Théorie de l'art moderne, p. 28.

48. P. Klee, Das bildnerische Denken, p. 82; Théorie de l'art moderne, p. 16.

49. Mot cité par D. Kahnweiler, Klee, Paris, Éditions Braun, 1950, p. 4.

50. Respectivement le texte "Wege des Naturstudiums» [«Voies diverses de l'étude de la nature»] et la conférence prononcée à Iéna connue sous le titre de sa première publication en 1945 Über die moderne Kunst. 
cette dernière ${ }^{51}$. L'art est d'abord une recherche des «racines», une plongée «dans les profondeurs [Urgrund] » ${ }^{52}$, en deçà de la nature donnée, du monde réel, vers des possibles du monde qu'il a pour tâche de faire apparaître. Ce qui rend possible la naissance d'un "nouveau naturel, le naturel de l'œuvre " 53 au sens d'une «résurrection de la nature» elle-même - et non d'une production attestant la puissance du sujet -, c'est un fondement commun à ces deux côtés, le sujet et l'objet: le Moi et le Toi selon l'expression de Klee ${ }^{54}$. Ce fondement commun est fourni par un nouveau couple, celui de la Terre et du Monde dont participent l'artiste et la nature, tous deux également traversés par leur polarité: il y a grâce à ces «chemins», entre l'artiste et l'objet,

un rapport par résonance qui transcende tout rapport optique. Premièrement, la voie d'un commun enracinement terrestre qui, d'en bas, gagne l'œil. Deuxièmement, la voie d'une commune participation cosmique qui survient d'en haut. Voies métaphysiques dans leur conjonction [...], la voie inférieure passe par l'ordre statique et produit des formes statiques, tandis que la voie supérieure passe par l'ordre dynamique 55 .

Toute apparition plastique aura ainsi lieu à partir de la tension de ces deux côtés, selon ce que Klee nomme une "mécanique plastique " ${ }^{6}$ : le côté terrestre qui est la dimension du repos, de la stabilité, de l'horizontalité, de la pesanteur; le côté cosmique qui est au contraire le mouvement, la verticalité, l'arrachement à la pesanteur ${ }^{57}$.

Il y a donc bien, à première vue, une analogie étonnante entre le propos de Klee et celui de Heidegger et, dès 1955, Will Grohmann se dit frappé de retrouver chez Heidegger ce que Klee a écrit trente ans plus tôt ${ }^{58}$. Cette analogie a une certaine consistance, dans la mesure où, d'abord, Terre et Monde apparaissent chez Klee comme chez Heidegger en quelque sorte

51. P. Klee, Das bildnerische Denken, p. 92; Théorie de l'art moderne, p. 28.

52. Ibid., p. $93 ;$ p. 31 .

53. Ibid., p. $67 ;$ p. 46.

54. Sur la tension entre «rêve, idée, imagination" et «formation" véritable, voir les notes de H.-F. Geist sur ses entretiens avec Klee, dans En souvenir de Paul Klee, A. Lampe (éd.), Paris, Éditions du Centre Pompidou, 2016, p. 57-68.

55. P. Klee, Das bildnerische Denken, p. 66-67; Théorie de l'art moderne, p. 45.

56. Ibid., p. $92 ;$ p. 27.

57. Ibid. Voir également: p. 67; p. 45.

58. Sans doute est-ce à la lecture de la conférence sur «La chose», publiée dans les Essais et conférences parus en 1954, que Grohmann se dit frappé par la convergence entre la pensée heideggerienne de la chose à partir du Geviert et le propos de Klee exposé trente ans auparavant: "Bien avant le philosophe Heidegger, Klee découvre donc une "quadrature" du moi et du toi, de la terre et de l'univers» (W. Grohmann, Paul Klee: 1879-1940, Paris, Flammarion, 1955, non paginé; voir également W. Grohmann, Paul Klee, Paris, Flincker, 1954, p. 181). 
comme les principes non subjectifs de l'émergence de l'œuvre et, à partir de là, des «choses » en général. Ensuite et surtout, ils sont de tels principes par leur seule corrélation, leur opposition et la tension entre eux que Heidegger désigne comme un " conflit [Streit]" 59 . Toutefois il faut aborder cette analogie avec une certaine circonspection : chez Klee, la distinction répond à une nécessité spécifique, celle de conceptualiser une difficulté de principe inhérente à l'art plastique. Elle paraît en outre se situer sur un plan avant tout "cosmologique» au sens d'un ordre et d'une articulation internes au monde et à son expérience, bref sur un plan «ontique». En revanche, chez Heidegger, d'une part la perspective est beaucoup plus large puisque cette distinction participe d'une caractérisation de la vérité même comme clivage entre "éclaircie» et "réserve " ${ }^{60}$; d'autre part l'un des deux termes du couple, le monde - dans tous les sens qu'il reçoit à partir d'Être et Temps -, non seulement est irréductible à une telle corrélation, mais implique toujours un plan ontologique, tant et si bien qu'il en vient quasiment à s'identifier à l'être en tant qu'il advient et se déploie historiquement, et ainsi à la "vérité de l'être». Les horizons de la réflexion sont donc bien différents de part et d'autre ${ }^{61}$. Cette référence à la détermination heideggerienne de la vérité nous conduit pourtant au seuil d'une seconde analogie, moins littéralement présente que celle marquée par le couple Terre-Monde mais sans doute d'une plus grande portée quant à la situation de l'œuvre de Klee dans l'histoire de l'être. Celle-ci repose sur la description de l'œuvre, chez Klee, en termes de processualité et d'historicité - à faible distance donc de «l'événement» de la vérité.

Nous avons rappelé la traversée des apparences finies que requiert le travail de l'artiste. Elle a pour conséquence que le séjour de l'artiste se trouve «dans le sein de la nature, dans le fonds primordial de la création où gît enfouie la clef de toute chose ${ }^{62}$. Dans ses notes, Heidegger reformule lapidairement ce propos de la conférence d’Iéna: «Habiter au cour de

59. Voir en particulier M. Heidegger, GA 5, 34 sqq.; Chemins..., p. 51 sqq.

60. Heidegger précise que «le monde n'est pas tout simplement l'ouvert correspondant à l'éclaircie; la terre n'est pas l'indécelable correspondant à la réserve»; il ajoute cependant que «la terre ne surgit à travers le monde, le monde ne se fonde sur la terre que dans la mesure où la vérité advient comme le combat originel entre éclaircie et réserve» (M. Heidegger, GA 5, 42; Chemins..., p. 60-61). Voir aussi les formulations de la première version de la conférence: M. Heidegger, Der Ursprung des Kunstwerkes, F.-W. von Herrmann (éd.), Francfort-sur-le-Main, V. Klostermann, 2012, p. 85 ; trad. fr.: De l'origine de l'œuvre d'art. Première version, C. Layet (trad.), Paris, Payot \& Rivages, 2014, p. 66.

61. Pour justifier sa thèse de la convergence des deux perspectives, $S$. Peetz doit la porter à un niveau de généralité où elle perd largement de sa substance. Voir S. Peetz, «Welt und Erde. Heidegger und Paul Klee», Études heideggeriennes, vol. 11, 1995, p. 167-187.

62. P. Klee, Das bildnerische Denken, p. 93; Théorie de l'art moderne, p. 30. 
la création [Im Herz der Schöpfung wohnen]» ${ }^{63}$. Il recopie également un extrait du journal de Klee: «j'habite aussi bien chez les morts que chez ceux qui ne sont pas encore nés. Un peu plus près du cœur de la création qu'à l'accoutumée» - à quoi Klee ajoute : «bien loin d'en être jamais assez proche! ${ }^{64}$. C'est également dans cette perspective qu'il faut saisir les deux formules les plus connues de Klee, elles aussi notées par Heidegger: «L'art ne reproduit pas le visible, il rend visible», et «Du modèle à la matrice! [Vom Vorbildichen zum Urbildlichen!] " ${ }^{65}$. En effet - contrairement à ce que paraît suggérer la seconde formule -, ce qu'il s'agit de «rendre visible» n'est pas à proprement parler une autre forme, une forme plus profonde ou plus vraie, mais quelque chose qui n'est plus de l'ordre de la forme. Au lieu de la «nature naturée», d'une «image finie de la nature», l'artiste doit selon Klee envisager la "nature naturante», c'est-à-dire «la création comme genèse " ${ }^{66}$. En d'autres termes, la forme elle-même doit paradoxalement être comprise "comme genèse, comme devenir» : la «forme $[$ Form $]$ » statique doit être mobilisée, et rapportée au processus de «formation [Formung] » qui lui est sous-jacent. Comme «fin », «mort», la forme doit être reconduite à la formation comme «mouvement, action", en un mot: à la «vie» ${ }^{67}$.

Cette perspective sur l'œuvre d'art y introduit de façon centrale la notion de temps, en opposition explicite avec la tradition issue de Lessing de séparation entre "arts du temps » et «arts de l'espace». Dès lors, en effet, que l'artiste cherche à épouser la mobilité même du processus créateur inhérent à la nature, son "horizon », dit Klee, s'élargit à partir du présent vers le passé, mais aussi «vers le futur » ${ }^{68}$. La forme véritablement plastique est justement celle par laquelle l'espace apparemment figé se mobilise et retrouve son unité avec le temps: la distinction entre espace et temps est en effet une "folie savante» que l'art doit surmonter en montrant combien "l'espace est aussi une notion temporelle " ${ }^{69}$. D'où l'importance que revêtent aux yeux de Klee le rythme et la ligne, conçus tous deux comme manifestations de l'unité originaire de l'espace et du temps ${ }^{70}$.

63. Voir G. Seubold, «Heideggers nachgelassene Klee-Notizen», p. 9.

64. Ibid., p. 8.

65. Voir respectivement P. Klee, Das bildnerische Denken, p. 76 et 93; Théorie de l'art moderne, p. 34 et 30 .

66. Ibid., p. $92 ;$ p. 28.

67. Ibid., p. $169 ;$ p. 60 .

68. Ibid., p. $92 ;$ p. $28-29$.

69. Ibid., p. $78 ;$ p. 37.

70. Voir notamment W. Grohmann, «Handzeichnungen von Paul Klee», in Texte zur Kunst der Moderne, K. Rudert, V. Billig (éd.), Munich, Hirmer, 2012, p. 137-141. 
Lorsque Klee affirme l'«identité du chemin et de l'œuvre [Identität von Weg und Werk] ${ }^{71}$, on comprend donc bien qu'il ne s'agit pas de rabattre l'œuvre sur le processus de sa fabrication, mais au contraire d'indiquer un certain mode d'être, qui est celui du devenir, du processus, voire de l'événement. La proximité est donc étroite avec le renversement opéré par Heidegger, dans sa conférence, de l'origine de l'œuvre d'art à l'œuvre comme origine - soit comme "avoir lieu» de la vérité [Geschehen der Wahrheit] -, c'est-à-dire aussi, plus généralement, avec la détermination de la vérité comme "avoir lieu». Il convient néanmoins, de nouveau, de circonscrire la portée d'un tel rapprochement. En décrivant aussi bien la nature que l'art sous l'horizon de l'inachèvement, du fragment appelant une infinie recréation, Klee laisse entrevoir l'ascendance romantique de sa pensée ${ }^{72}$ : en subordonnant l'être au devenir, Klee n'en reconduit pas moins une ontologie traditionnelle de la présence. Ainsi, le mode d'être apparaissant dans l'œuvre, telle qu'elle est conçue par Klee, ne consonne pas entièrement avec la tentative de Heidegger de penser l'être à partir de l'événement ${ }^{73}$.

C'est ici que vient jouer l'hiatus pointé par Heidegger entre les textes et les œuvres de Klee. L'«auto-interprétation» de Klee lui paraît en effet «erronée». L'erreur procéderait, selon Heidegger, de la situation de cette auto-interprétation dans l'espace propre à la «topologie de l'être», en l'occurrence «à l'un des points de contacts les plus périlleux entre la métaphysique et ce qui est encore à venir ${ }^{74}$. Dans cette situation charnière, l'artiste Klee aurait mis en œuvre une mutation que le penseur Klee n'aurait pas été à même de saisir et d'énoncer. Le parallèle est ici tentant avec Hölderlin, dont Heidegger dit qu'il «pense encore métaphysiquement. Mais il poétise autrement $»^{75}$. Tant et si bien qu'il peut être désigné - Heidegger le fait à plusieurs reprises - comme "le poète de l'autre commencement ${ }^{76}$. $\mathrm{Au}$ fil de cette analogie, il serait ainsi loisible de voir en Klee le peintre de l'autre commencement, qui aurait ainsi devancé le penseur Klee.

Sans se prononcer sur la pertinence d'une telle formule, on peut néanmoins redire en sa faveur que c'est bien la question du dépassement

71. P. Klee, Das bildnerische Denken, p. 169; Théorie de l'art moderne, p. 60.

72. Voir D. Kahnweiler, Klee, p. 10-12.

73. L'attention de Merleau-Ponty à la «vie» comme telle le conduit à mettre particulièrement en exergue, à partir de Klee, la "générativité» de l'œuvre, et notamment la ligne comme "épure d'une genèse des choses» (voir L'œil et l'esprit, Paris, Gallimard, 1964, chap. 4, p. 61-87).

74. H. W. Petzet, Le chemin de l'étoile..., p. 172.

75. M. Heidegger, Hölderlins Hymne "Andenken", GA 52, 120.

76. Voir M. Heidegger, Besinnung, GA 66, 426, et Über den Anfang, GA 70, 160. 
de la métaphysique qui fait de la rencontre entre Klee et Heidegger une véritable rencontre, au sens où Klee intervient dans ou sur la pensée de Heidegger. Ce qui nous amène à souligner, en guise de conclusion, deux points. Tout d'abord, Heidegger, dans les années 1930, présente la pensée, la politique et l'art comme les trois "puissances» susceptibles de donner une configuration au monde historique. Néanmoins, il lui arrive de donner la préséance à l'art et à son pouvoir de commencement propre 77 . Cette sorte de privilège accordé à l'art trouve à s'exercer pleinement lorsque c'est l'art lui-même qui est en cause. Ainsi, Heidegger demande: «Que peut être l'art à l'époque du dispositif, et comment peut-il l'être?». Cela ne peut être décidé, écrit-il, "qu'“artistiquement", si bien que la réponse à cette question réside dans cet art même, et là seulement». "Über Kunst

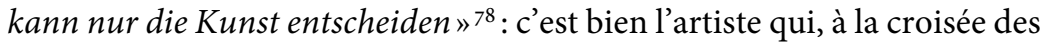
chemins de l'histoire de la métaphysique, décide de la voie à emprunter. Or, précisément, avec Klee, "l'art accomplit une mutation [die Kunst sich wandle] ${ }^{79}$ : et c'est bien l'artiste qui indique - plastiquement - une possibilité non métaphysique, à laquelle il conviendrait que la pensée réponde sur ce même mode non métaphysique.

On peut bien évidemment s'interroger sur la réalité de cette intervention de l'art dans ou sur la philosophie: Heidegger, incontestablement, aborde et interprète Klee à partir d'une perspective qui est la sienne propre, celle de l'histoire de l'être. Cette intervention nous paraît pourtant attestée par l'évocation d'une «seconde partie» de la conférence sur l'œuvre, à condition d'en bien comprendre le sens. Car la fonction qui revient potentiellement à cette seconde partie ne réside pas, contrairement à ce que disent les commentateurs, dans une sorte de mise à jour, par laquelle le propos deviendrait également valable pour l'art moderne (Peetz), ou plus exactement pour un art adapté au monde technique (Pöggeler). Elle devrait plutôt assumer une fonction herméneutique, celle de dénouer les tensions internes à une conférence qui était tout à la fois encore métaphysique et déjà post-métaphysique. En effet, la possibilité d'une lecture non métaphysique de la conférence avait un temps paru se refermer; or la rencontre avec Klee permet de rouvrir cette possibilité. Et c'est tout le sens du «Supplément» de 1956, contemporain de cette rencontre, d'insister sur cette possibilité. Dans ses premières lignes, il reconnaît la tension qui

77. C'est notamment ce que suggèrent les dernières pages de la conférence sur l'œuvre d'art, qui évoquent la mise en mouvement de l'histoire par le «choc» de l'art. Voir M. Heidegger, GA 5, 65-66; Chemins..., p. 87-89. Voir également une note contemporaine de la conférence (1934-1935) dans GA 94, 216.

78. M. Heidegger, "Kunst und Technik», in GA 76, 378-379.

79. H. W. Petzet, Le chemin de l'étoile..., p. 172. 
règne dans la conférence entre «Feststellen der Wahrheit [constituer la vérité] » et «Geschehenlassen der Ankunft der Wahrheit [laisser avoir lieu l'arrivée de la vérité $]{ }^{80}$. Mais l'ensemble du «Supplément» insiste sur la possibilité de lever cette contradiction, et fait porter tout le poids sur «ce qui vient». Ainsi, le rapport traditionnel entre art et philosophie s'inverse; et c'est bien une œuvre plastique, celle de Klee, qui paraît indiquer en quel sens il convient de lire un texte philosophique.

Guillaume FAGNIEZ 
